\title{
Limites duais para o problema integrado de dimensionamento e sequenciamento de lotes modelado com restrições multicommodity
}

Tiago Tiburcio da Silva ${ }^{1}$

Socorro Rangel ${ }^{2}$

Departamento de Matemática Aplicada

R. Cristóvão Colombo, 2265. Jd. Nazareth, São José do Rio Preto, Brazil, 15054000

\begin{abstract}
Resumo. Neste trabalho é discutido o problema integrado de dimensionamento e de sequenciamento de lotes utilizando um modelo matemático que exclui subsequências desconexas através de restrições de fluxo multicommodity. Propomos limites primais a partir de uma heurística gulosa e limites duais baseados na relaxação Lagrangeana e na relaxação Lagrangeana/Surrogate. Os problemas duais associados são resolvidos usando o Algoritmo de Subgradiente e o Algoritmo de Volume. O método que obteve melhor desempenho foi o dual lagrangeano resolvido pelo Algoritmo do Subgradiente.
\end{abstract}

Palavras-chave. dimensionamento, sequenciamento, relaxação Lagrangeana

\section{Introdução}

As decisões do planejamento da produção no setor de manufatura envolvem, entre outros, determinar o uso mais eficiente dos recursos para atender a demanda dos itens requeridos pelos clientes. Estas decisões são tomadas geralmente em duas etapas: uma etapa de dimensionamento dos lotes e outra de sequenciamento dos lotes [2]. Na etapa de dimensionamento deseja-se determinar quanto de cada item produzir (tamanho dos lotes) a fim de atender as demandas pré-especificadas, sob as condições e capacidades operacionais existentes. Na etapa de sequenciamento deseja-se ordenar a produção dos lotes [15]. Se essas decisões são tomadas de forma independente, podem ocorrer infactibilidades ou um menor aproveitamento da capacidade de produção, daí o interesse em integrar essas duas decisões. O Problema Integrado de Dimensionamento e Sequenciamento de lotes (PIDS) consiste em determinar quanto de cada item produzir e em que ordem, de modo a atender as demandas previstas com o objetivo de reduzir custos e tempos de produção, ou seja, determinar simultaneamente a quantidade a ser produzida em cada máquina, em cada

\footnotetext{
${ }^{1}$ ttsilva@gmail.com

${ }^{2}$ socorro@ibilce.unesp.br
} 
período e em que ordem. Existem muitos trabalhos na literatura sobre o PIDS que incluem modelagem e métodos de solução. Revisões bibliográficas podem ser encontradas por exemplo em [7] e [9]. Na literatura, o modelo GLSP, em que o sequenciamento é obtido pela divisão dos períodos em subperiodos menores, é usado para representar o PIDS em diferentes contextos industriais, alguns exemplos são: bebidas [4], alimentação animal [14], fundições [1].

Neste trabalho, propomos limites primais e duais para o Modelo PIDS-MCF proposto para o PIDS em [12]. Neste modelo, as decisões e restrições associadas ao sequenciamento dos lotes são baseadas nas restrições de eliminação de subrotas tipo multicommodity para o Problema do Caixeiro Viajante. Em que pese o fato da formulação PIDS-MCF ser mais forte que a formulação baseada no GLSP, a solução de instâncias do modelo usando o método branch and cut incluido em solvers comerciais é cara computacionalmente [10]. Há portanto, o interesse no desenvolvimento de métodos eficientes para obter limites primais e duais para esta formulação, explorando assim, a sua qualidade teórica de forma também computacional, o que é importante para as aplicações industriais. Este trabalho contribui nesta direção. O texto está organizado da seguinte forma: na Seção 2 definimos o problema e apresentamos o modelo matemático PIDS-MCF, na Seção 3 descrevemos os métodos para obter os limites primais e duais, na Seção 4 apresentamos os resultados computacionais e, na Seção 5 as considerações finais.

\section{Definição do problema e Modelos Matemáticos}

Neste trabalho consideramos um processo produtivo em que um conjunto de $J$ itens deve ser produzido em uma única máquina e em $T$ períodos. A demanda de cada item em cada periodo é conhecida e a capacidade da máquina em cada período é limitada. $\mathrm{O}$ tempo de preparo da máquina para a produção de um novo item é relevante e dependente da sequência de produção. No cenário considerado, também é permitido o atraso no atendimento da demanda sob uma penalização. O problema integrado de dimensonamento e sequenciamento de lotes consiste então em determinar a quantidade a ser produzida de cada item em cada período e a ordem em que os itens serão produzidos, com o objetivo de minimizar o custo total da produção em termos de custos de estoque, atraso e trocas.

Para representar este problema, vamos considerar o modelo proposto em [12]. Para descrevê-lo, usaremos os índices $i, j=1 \ldots J$ para representar os itens e $t=1 \ldots T$ para representar os períodos. Os parâmetros usados são: $h_{j}$ custo de estoque, $g_{j}$ custo de atraso,$s_{i j}$ custo de troca, $p_{j}$ tempo de produção, $b_{i j}$ tempo de troca, $d_{j t}$ demanda, $C_{t}$ capacidade da máquina. As decisões são representadas pelas incógnitas: $x_{j t}$ tamanho do lote, $I_{j t}^{+}$quantidade em estoque, $I_{j t}^{-}$quantidade em atraso, $z_{i j t}=1$ define se há troca, e $w_{j}=1$ se a máquina está preparada. O modelo PIDS-MCF é dados pelas expressões (1)-(10).

$$
\text { Min } \mathrm{Z}=\sum_{j \in J} \sum_{t \in T}\left(h_{j} I_{j t}^{+}+g_{j} I_{j t}^{-}\right)+\sum_{t \in T} \sum_{i \in J} \sum_{\substack{j \in J \\ j \neq i}} s_{i j} z_{i j t}
$$

sujeito a: 
Restrições associadas ao dimensionamento de lotes

$$
\begin{aligned}
& I_{j(t-1)}^{+}+I_{j t}^{-}+x_{j t}-I_{j t}^{+}-I_{j(t-1)}^{-}=d_{j t}, \quad \forall j, \forall t \\
& \sum_{j \in J} p_{j} x_{j t}+\sum_{i \in J} \sum_{\substack{j \in J \\
j \neq i}} b_{i j} z_{i j t} \leq C_{t}, \\
& x_{j t} \leq \frac{C_{t}}{p_{j}} w_{j t}, \\
& w_{j t}=\sum_{\substack{i=i_{0} \\
i \neq j}}^{J} z_{i j t}, \\
& \forall t \\
& \forall j, \forall t \\
& \forall j, \forall t
\end{aligned}
$$

Restrições associadas ao sequenciamento de lotes

$$
\begin{gathered}
\sum_{j \in J} z_{i_{0} j t} \geq \sum_{i=i_{0}}^{J} z_{i k t}, \\
\sum_{\substack{i=i_{0} \\
i \neq k}}^{J} z_{i k t}=\sum_{\substack{j=i_{0} \\
j \neq k}}^{J} z_{k j t}, \\
\sum_{\substack{j=i_{0} \\
j \neq i}}^{J} z_{i j t} \leq 1,
\end{gathered}
$$$$
\forall k \in J ; k \neq i, \forall t
$$$$
\forall k \in J, \forall t
$$$$
\forall i=i_{0}, 1, \ldots, J, \forall t
$$

Restrições para eliminação de subsequencias desconexas

$$
x_{j t}, I_{j t}^{+}, I_{j t}^{-} \geq 0, I_{j 0}^{+}=I_{j 0}^{-}=0, w_{j t}=0 / 1, \forall j, \forall t ; \quad z_{i j t}=0 / 1, \forall i, j, \forall t .
$$

A função objetivo (1) minimiza o custo total medido em termos dos custos de estoque, atraso e troca entre os itens. As restrições (2) garanten o atendimento da demanda permitindo atrasos. As restrições (3) garantem que o tempo de produção e de troca entre os itens não ultrapasse a capacidade da máquina. As restrições (4) e (5) garantem que só haverá produção se a máquina estiver preparada. A restrição (5) é redundante, porém a sua inclusão no modelo diminui o tempo total de resolução [13]. As restrições (6) - (8) garantem que todos os itens produzidos serão sequenciados. O domínio das incógnitas é definido em (10). Neste modelo é incluído um item fantasma $i_{0}$ com custo de troca associado igual a zero e tal que $\sum_{k=1}^{J} z_{i_{0} k t}=1, \forall t$ (isto é, $i_{0}$ é o primeiro item da sequência de cada período).

Existem diversas alternativas para modelar as restrições $(9)$ (e.g. $[7,10])$. No modelo PIDS-MCF são usadas as restrições $(11)$ - (14). Neste modelo é considerada uma rede com $J+1$ vértices e que existe uma oferta de $J$ commodities (itens) no vértice $i_{0}$ e uma demanda de uma unidade da commodity $r=1 \ldots J$ no vértice $r$. Uma nova incógnita é necessária, e em termos do sequenciamento, se $m_{r i j t}=1$ temos que o item $r$ é incluído na sequência, e então o item $i$ é produzido antes do item $j$.

$$
\sum_{j \in J} m_{r i_{0} j t}-\sum_{j \in J} m_{r j i_{0} t}=\sum_{\substack{j=i_{0} \\ j \neq r}}^{J} z_{j r t}, \quad \forall r, \forall t
$$




$$
\begin{aligned}
& \sum_{j=i_{0}}^{J} m_{r j r t}-\sum_{j=i_{0}}^{J} m_{r r j t}=\sum_{j=i_{0}}^{J} z_{j r t}, \quad \forall r, \forall t \\
& \sum_{i=i_{0}}^{J} m_{\text {rijt }}=\sum_{i=i_{0}}^{J} m_{r j i t}, \quad \forall r, \forall t ; j \neq r, \forall t \\
& 0 \leq m_{\text {rijt }} \leq z_{i j t}, \quad \forall i, j=i_{0}, 1, \ldots, J, \forall r, \forall t .
\end{aligned}
$$

As restrições (11) e (12) são impostas apenas se a máquina estiver preparada para a produção do item $r$ e garantem que se este item for incluído na sequência, pelo menos um outro também deve ser incluído. As restrições (13) garantem a conservação do fluxo no vértice $r$ para todos os itens $j \neq r$ e as restrições (14) garantem que o item $i$ é produzido antes do item $j$ na sequência que inclui o item $r$ apenas se houver troca do item $i$ para o item $j$.

\section{$3 \quad$ Limites primais e duais}

Para se obter uma solução factível para o PIDS-MCF (limite primal) foi desenvolvida uma heurística gulosa baseada na seguinte ideia. Passo 1: ordena-se os itens de forma não decrescente do custo de troca. Passo 2: Resolve-se o problema de dimensionamento de lotes (formado pelas expressões (1), (2)-(5), e (10) descritas na Seção 2), com as variáveis de troca fixadas de acordo com a ordem definida no Passo 1.

Para se obter limites duais para o PIDS-MCF foram desenvolvidos dois tipos de relaxação. A Relaxação Lagrangeana [6] é obtida dualizando as restrições (3) com o multiplicador $\pi^{1} \in \mathbb{R}_{+}^{T}$ e (5) com o multiplicador $\pi^{2} \in \mathbb{R}_{+}^{J T}$. O subproblema Lagrangeano é dado por (15)-(16).

$$
\begin{array}{r}
\operatorname{Min} \mathrm{L}\left(\mathrm{x}, I^{+}, I^{-}, \mathrm{z}, \pi^{1}, \pi^{2}\right)=\sum_{j \in J} \sum_{t \in T}\left(h_{j} I_{j t}^{+}+g_{j} I_{j t}^{-}\right)+\sum_{t \in T} \sum_{\substack { i \in J \\
\begin{subarray}{c}{j \in J \\
j \neq i{ i \in J \\
\begin{subarray} { c } { j \in J \\
j \neq i } }\end{subarray}} s_{i j} z_{i j t} \\
+\sum_{t \in T} \pi_{t}^{1}\left(\sum_{j \in J} p_{j} x_{j t}+\sum_{\substack { i \in J \\
\begin{subarray}{c}{j \in J \\
j \neq i{ i \in J \\
\begin{subarray} { c } { j \in J \\
j \neq i } }\end{subarray}} b_{i j} z_{i j t}-C_{t}\right)+\sum_{j \in J} \sum_{t \in T} \pi_{j t}^{2}\left(w_{j t}-\sum_{\substack{i=i_{0} \\
i \neq j}}^{J} z_{i j t}\right) \\
\text { sujeito a: (2), (4),(6)-(8),(10), (11)-(14) }
\end{array}
$$

Para obter a Relaxação Lagrangeana/Surrogate, inicialmente construimos a restrição surrogate (17) a partir das restrições (5) com o multiplicador Surrogate $\pi^{2} \in \mathbb{R}_{+}^{J T}$.

$$
\sum_{j \in J} \sum_{t \in T} \pi_{j t}^{2}\left(w_{j t}-\sum_{\substack{i=i_{0} \\ i \neq j}}^{J} z_{i j t}\right)=0 .
$$


O subproblema Lagrangeano/Surrogate é obtido dualizando a restrição surrogate com o multiplicador de Lagrange $t$, e as restrições (3) com o multiplicador $\pi^{1} \in \mathbb{R}_{+}^{T}$, obtendo o subproblema descrito pelas expressões (18)-(19):

$$
\begin{array}{r}
\operatorname{Min} \operatorname{LS}\left(\mathrm{x}, I^{+}, I^{-}, \mathrm{z}, \pi^{1}, \pi^{2}\right)=\sum_{j \in J} \sum_{t \in T}\left(h_{j} I_{j t}^{+}+g_{j} I_{j t}^{-}\right)+\sum_{t \in T} \sum_{i \in J} \sum_{\substack{j \in J \\
j \neq i}} s_{i j} z_{i j t} \\
+\sum_{t \in T} \pi_{t}^{1}\left(\sum_{j \in J} p_{j} x_{j t}+\sum_{\substack { i \in J \\
\begin{subarray}{c}{j \in J \\
j \neq i{ i \in J \\
\begin{subarray} { c } { j \in J \\
j \neq i } }\end{subarray}} b_{i j} z_{i j t}-C_{t}\right)+\sum_{j \in J} \sum_{t \in T} t \pi_{j t}^{2}\left(w_{j t}-\sum_{\substack{i=i_{0} \\
i \neq j}}^{J} z_{i j t}\right) \\
\text { sujeito a: (2), (4),(6)-(8),(10), (11)-(14) }
\end{array}
$$

É interessante observar que tanto o subproblema lagrangeano quanto o subproblema lagrangeano/surrogate podem ser decompostos em dois: um problema de dimensionamento de lotes não-capacitado e um problema de sequenciamento. Para obter os limitantes duais associados utilizamos os algoritmos de subgradiente [8] e de volume [3]. Mais detalhes sobre a heurística gulosa e os algoritmos usados para resolver os problemas duais podem ser obtidos em [13].

\section{Resultados computacionais}

Os modelos e os algoritmos foram escritos usando a sintaxe da linguagem de modelagem AMPL [5] e os problemas de otimização associados foram resolvidos pelo sistema IBMCplex 12.6. Os testes foram executados em um computador com processador Intel Core i7 com $3.40 \mathrm{GHz}$ e com $12 \mathrm{~GB}$ de memória RAM, sob a plataforma Windows 7.

Para avaliarmos os limitantes propostos utilizamos 15 exemplares considerando $J=25$ itens, propostos em [10]. Os exemplares foram divididos em 3 classes de acordo com o número de períodos (cada classe com 5 exemplares) sendo $t=5,10$, e 15 para as classes 1, 2, e 3 respectivamente. Foram realizados dois testes fixando o tempo máximo de CPU em 10 minutos, e em uma hora. Para comparar a qualidade dos limitantes duais (LD) usamos como referência os limites primais (LP) obtidos com as soluções factíveis fornecidas pela heurística gulosa $(g)$ e pelo Cplex $(c)$. Para cada exemplar, foi calculada a diferença relativa: $\mathrm{GAP}_{i}^{j}=\left(L P_{i}-L D^{j}\right) / L P_{i}$, em que $i \in\{g, c\}$ e $j \in\{S, V, S U\}$ ( $S$ :para o valor do dual Lagrangeano resolvido pelo Método de Subgradiente; $V$ : para o valor do dual Lagrangeano resolvido pelo algoritmo de volume; $S U$ : para o valor do dual LagrangeanoSurrogate resolvido pelo Método de Subgradiente). A Tabela 1 exibe o valor médio de cada uma dessas medidas para cada classe de instâncias considerando o limite primal obtido pela heurística gulosa e a Tabela 2 exibe os resultados considerando o limite primal obtido pelo Cplex no tempo limite de uma hora. Para as Classes 2 e 3 não foi possível calcular $\operatorname{GAP}_{i}^{V}$ e $\operatorname{GAP}_{i}^{S U}$. No tempo limite de uma hora os algoritmos não convergiram.

Analisados os resultados referentes ao tempo de 10 minutos, para a classe 1 , o dual lagrangeano/surrogate teve melhor desempenho, em particular quando o GAP é calculado relativo à melhor solução Cplex (Tabela 2). Para um tempo maior de execução, nota-se 
pouca evolução do algoritmo subgradiente usado na solução do dual lagrangeano. Porém para os outros dois métodos há uma considerável melhoria. Mais detalhes sobre o estudo computacional podem ser encontrados em [13].

\begin{tabular}{|c|c|c|c||c|c|c|}
\hline \multirow{2}{*}{ Classe } & \multicolumn{3}{|c||}{$10 \mathrm{~min}$} & \multicolumn{3}{c|}{1 hora } \\
\cline { 2 - 7 } & $\mathrm{GAP}_{g}^{S}$ & $\mathrm{GAP}_{g}^{V}$ & $\mathrm{GAP}_{g}^{S U}$ & $\mathrm{GAP}_{g}^{S}$ & $\mathrm{GAP}_{g}^{V}$ & $\mathrm{GAP}_{g}^{S U}$ \\
\hline 1 & $30.21 \%$ & $52.91 \%$ & $21.24 \%$ & $30.15 \%$ & $25.36 \%$ & $12.56 \%$ \\
2 & $29.49 \%$ & - & - & $27.75 \%$ & - & - \\
3 & $35.01 \%$ & - & - & $27.11 \%$ & - & - \\
\hline
\end{tabular}

Tabela 1: Gap relativo médio referente à solução heurística

\begin{tabular}{|c|c|c|c||c|c|c|}
\hline \multirow{2}{*}{ Classe } & \multicolumn{3}{|c||}{10 min } & \multicolumn{3}{c|}{1 hora } \\
\cline { 2 - 7 } & $\mathrm{GAP}_{c}^{S}$ & $\mathrm{GAP}_{c}^{V}$ & $\mathrm{GAP}_{c}^{S U}$ & $\mathrm{GAP}_{c}^{S}$ & $\mathrm{GAP}^{V}$ & $\mathrm{GAP}_{c}^{S U}$ \\
\hline 1 & $22.03 \%$ & $47.41 \%$ & $12.06 \%$ & $21.96 \%$ & $16.61 \%$ & $2.32 \%$ \\
2 & $18.36 \%$ & - & - & $15.28 \%$ & - & - \\
3 & $21.85 \%$ & - & - & $12.33 \%$ & - & - \\
\hline
\end{tabular}

Tabela 2: Gap relativo médio referente à melhor solução CPLEX

\section{Considerações finais}

Neste trabalho, propusemos limites duais para o problema PIDS formulado com restrições de fluxo multicommodity baseados na dualização das restrições de capacidade e da restrição de acoplamento dentre as decisões de preparo e de troca. Os problemas duais associados às relaxações Lagrangeana e Lagrangeana/Surrogate foram resolvidos usando a algoritmo de subgradientes e o de volume. Os resultados obtidos foram comparados usando como medida a diferença entre os limites duais e o valor da solução factivel obtida por uma heurística gulosa e pelo Cplex. O estudo computacional realizado com instâncias geradas aleatoriamente indicam que o dual lagrangeano resolvido pelo Algoritmo de Subgradiente apresentou melhor desempenho considerando que foi o único método capaz de convergir no tempo limite de execução para as três classes de intâncias testadas. Para melhoria da convergência dos métodos, trabalhos futuros serão realizados utilizando técnicas de estabilização da solução dual. O uso do algoritmo de subgradiente projetado também pode contribuir para aumentar a eficiência do método.

\section{Agradecimento}

Agradecemos ao apoio do CNPq, CAPES, e FAPESP. A segunda autora agradece a hospitalidade da Universidad de Valladolid onde parte deste trabalho foi desenvolvido.

\section{Referências}

[1] S. A. Araujo, M. N. Arenales, A. R. Clark. Lot sizing and furnace scheduling in small foundries, Computers \& Operations Research, 35(3), 916 - 932, 2008. 
[2] S. A. Araujo, S. Rangel. Matemática Aplicada ao Planejamento da Produção e Logística, Notas em Matemática Aplicada, v. 76, 2014.

[3] F. Barahona, R. Anbil. The volume algorithm: producing primal solutions with a subgradient method, Mathematical Programming, 87(3) : 385 - 389, 2000.

[4] D. Ferreira, R. Morabito, S. Rangel. Solution approaches for the soft drink integrated production lot sizing and scheduling problem, European Journal of Operational Research, 196(2), 697 - 706, 2009.

[5] R. Fourer, D. M. Gay, B. W. Kernighan, AMPL - A Modeling Language for Mathematical Programming, Second Edition, Cengage Learning, 2002, ISBN 0-534-38809-4.

[6] M. Guignard. Lagrangean Relaxation, Top, Volume 11, Number 2, 151 - 228, December 2003 .

[7] L. Guimarães, D. Klabjan, B. Almada-Lobo. Modeling lotsizing and scheduling problems with sequence dependent setups. European Journal of Operational Research, 239 (3), 644-662, 2014.

[8] A. F. Izmailov, M. V. Solodov. Otimização - Vol. 2. Métodos Computacionais, IMPA, Rio de Janeiro, 2005.

[9] R. Jans, Z. Degraeve. Modeling Industrial Lot Sizing Problems: A Review, International Journal of Production Research, 46(6), 1619 - 1643, 2008.

[10] M. Maldonado. Reformulações para o Problema Integrado de Dimensionamento e Sequenciamento da Produção, Tese de Doutorado, UNESP/IBILCE, 2015.

[11] M. G. Narciso. A relaxação Lagrangeana/Surrogate e algumas aplicações em otimização combinatória. Tese de Doutorado em Computação Aplicada, INPE, 1998.

[12] S. Rangel, M. Maldonado. Three Mathematical Models for a Integrated Lot Sizing and Scheduling Problem. Proceeding Series of the Brazilian Society of Applied and Computational Mathematics, vol. 2, n. 1, 2014. DOI: http://dx.doi.org/10.5540/03.2014.002.01.0117.

[13] T. T. Silva. Um estudo sobre limites duais para o problema integrado de dimensionamento de lotes e sequenciamento da produção, Dissertação de Mestrado, UNESP/IBILCE, 2015.

[14] E. A. V. Toso, R. Morabito, A. R. Clark. Lot sizing and sequencing optimisation at an animal-feed plant, Computers \& Industrial Engineering, 57(3), 813 - 821, 2009.

[15] C. Wolosewicz, S. Dauzère-Pérès, R. Aggoune, R. A Lagrangian heuristic for an integrated lot-sizing and fixed scheduling problem, European Journal of Operational Research, 244(1), 3-12, 2015. 